\title{
« La force, qui trace les frontières, est-elle habile à disposer des peuples?"
}

\author{
Guerre et histoire dans le théâtre d'André Ricard
}

"Can Force, that Defines Borders, also Dispose of Peoples?"

War and History in the Theatre of André Ricard

\section{'La fuerza, que delimita las fronteras, ¿tiene capacidad para disponer de los pueblos?'}

\section{Guerra e historia en el teatro de André Ricard}

\section{Lucie Robert}

Volume 37, numéro 2 (110), hiver 2012

La guerre dans la littérature québécoise

URI : https://id.erudit.org/iderudit/1008578ar

DOI : https://doi.org/10.7202/1008578ar

\section{Aller au sommaire du numéro}

\section{Éditeur(s)}

Université du Québec à Montréal

\section{ISSN}

0318-9201 (imprimé)

1705-933X (numérique)

Découvrir la revue

Citer cet article

Robert, L. (2012). « La force, qui trace les frontières, est-elle habile à disposer des peuples ? " : guerre et histoire dans le théâtre d'André Ricard. Voix et Images, 37(2), 95-107. https://doi.org/10.7202/1008578ar

\section{Résumé de l'article}

Parle-t-on de la même manière d'une guerre coloniale, d'une guerre civile et d'une guerre mondiale ? D'une guerre qui se déroule ici et d'une guerre qui se déroule ailleurs ? Chaque guerre a ses enjeux propres, en effet, et chacune reconfigure autrement l'espace de son origine et celui de sa résolution. Je prendrai ici pour exemple la trilogie d'André Ricard qui les aborde toutes : la guerre de Sept Ans, qui s'est soldée par l'abandon de la Nouvelle-France ; la guerre civile qu'ont failli devenir les deux rébellions de 1837 et 1838 ; la Deuxième Guerre mondiale, à laquelle se sont opposés massivement les Canadiens français, bien que des bataillons de volontaires y aient participé. Écrite et publiée sur vingt-cinq ans, la trilogie, composée de La longue marche dans les Avents, du Tréteau des apatrides et de Gens sans aveu, porte aussi en elle les marques de l'histoire contemporaine du théâtre comme celles de la démarche singulière d'un auteur dramatique peu joué et dont l'écriture conquiert progressivement son autonomie en regard de la scène. Ricard nous convie à une relecture de l'histoire du Québec, où chaque guerre reconfigure l’identité nationale. 


\section{"LA FORCE, Q U I TRACE \\ LES FRONTIÈRES, EST-ELLE HABILE \\ À DISPOSER DES PEUPLES 1 ? "}

Guerre et histoire dans le théâtre d'André Ricard

$+\quad+$

\section{LUCIE ROBERT}

Centre de recherche interuniversitaire sur la littérature et la culture québécoises (CRILCQ),

Université du Québec à Montréal

Deux grandes inventions marquent le début de notre civilisation:

la poudre à canon et l'imprimerie.

La guerre et les livres ${ }^{2}$.

Locales ou étrangères, mondiales ou civiles, les guerres semblent avoir en commun de désigner un conflit pour l'occupation du territoire, que celui-ci soit défini par des frontières légitimes ou réclamé comme terre ancestrale. L'existence de frontières à défendre, y compris quand celles-ci ont été imposées par la force, paraît rendre plus honorable, ou du moins plus organisée, la violence entre les hommes et l'exercice du pouvoir qui en découle. En effet, la reconnaissance internationale des frontières donne toujours une certaine légitimité aux pouvoirs établis et elle rend précaire le statut des peuples qui, à tort ou à raison, se croient spoliés de leur territoire. Toutefois, doit-on limiter la notion de guerre aux querelles de frontières ${ }^{3}$ ? Car l'on pense généralement la guerre à travers le prisme de l'Europe, c'est-à-dire, pour le Xx siècle, en se référant à la Première puis à la Deuxième Guerre mondiale comme à des guerres de résistance légitime à l'envahisseur étranger. Mais est-ce encore une guerre que la guerre civile, comme en Espagne ou au Liban, c'est-à-dire une guerre intraterritoriale qui vise la soumission d'un groupe d'habitants à un autre, voire son expulsion hors du territoire? Et est-ce une guerre civile qu'une rébellion, une insurrection ou un soulèvement populaire? Ceux-ci sont évidemment d'échelle plus petite, mais ils désignent quand même une guerre civile écrasée tôt dans son développement. Les considérations de frontières sont ce qui fonde normalement l'idée de guerre en tant que conflit pour l'occupation d'un espace donné, bien qu'elles mettent davantage en valeur son caractère indécent, comme s'il s'agissait d'un jeu que d'aucuns prendraient trop au sérieux. Entre l'absurde ainsi révélé et l'humanité blessée, les écrivains s'interrogent: n'y a-t-il pas que

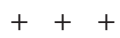

1 André Ricard, Le tréteau des apatrides ou La veillée en armes. Théâtre, introduction de Lucile Martineau, Québec, Septentrion, 1995, p. 211. 2 Lise Vaillancourt, Marie-Antoine, opus I, Montréal, Les Herbes rouges, coll. "Thêâtre", 1988, p. 62. 3 Voir Yves Lacoste, La géographie, ça sert d'abord à faire la guerre, Paris, François Maspero, 1976, $187 \mathrm{p}$. 
l'expérience de la guerre change quelqu'un et que, la guerre étant un combat collectif, son expérience change aussi la collectivité ? De cette manière, la guerre se fait expérience de la violence, oui, mais aussi expérience de la différence dans la relation avec l'ennemi; elle se fait mesure de la civilisation dans le sort réservé au vaincu; elle se fait choix moral autant que politique dans la reconstruction d'une société d'aprèsconflit.

Parmi ces écrivains qui parlent de la guerre, il faut sans doute faire un sort particulier aux auteurs dramatiques, car le thêâtre est né de la guerre ${ }^{4}$; et s'il est vrai, comme l'affirme Anne Ubersfeld, que «la structure de presque tous les récits dramatiques peut se lire comme un conflit d'espaces, ou comme la conquête ou l'abandon d'un certain espace $^{5}$ ", $c^{\prime}$ est qu'il la porte dans sa nature même ${ }^{6}$. Toutefois, peut-on représenter la guerre sans tomber dans l'obscénité ${ }^{7}$ ? En parler, certainement, mais la mettre en scène, la donner à voir et à entendre? À vrai dire, ils sont rares les auteurs dramatiques qui ont mis la guerre en scène. En se plaçant du point de vue des vaincus (les Perses), en particulier du point de vue des femmes et des vieillards qui attendent des nouvelles du front, Eschyle, déjā, montrait plutôt les mécanismes guerriers que la guerre elle-même ${ }^{8}$. On opposera alors l'idée d'une dramaturgie de la guerre à celle d'une dramaturgie de l'état de guerre ${ }^{9}$ et l'on observera que, dans le thêâtre contemporain qui a renoncé depuis longtemps au conflit dramatique tel que l'entendait Aristote, l'on ne trouve guère de dramaturgie de la guerre proprement dite. Il n'y a en effet aucune gloire littéraire à mettre en scène la victoire de l'État, et encore moins celle du chef de l'État. Le triomphe du plus fort n'est jamais un bon ressort dramatique. C'est plutôt là la matière de l'éloge ou de la poésie de circonstances. Ainsi, la dramaturgie québécoise accorde bien peu de place aux guerres américaines, celle de 1775 ou celle de 1812, pour laquelle nous n'avons que cette discrète référence au personnage héroïque de Laura Secord, mais sur le mode ironique propre à Claude Roussin ${ }^{10}$. Elle est plus diserte sur les guerres civiles du XIXe siècle et sur les deux guerres mondiales du Xxe siècle, chacune étant vécue d'abord comme la défaite du Canada français, opposé deux fois à la conscription, puis comme source de conflits sociaux dans un après-guerre marqué par le retour des conscrits, ce que montrent aussi bien Tit-Coq (1948) de Gratien Gélinas qu'Un simple soldat (1957) de Marcel Dubé ${ }^{11}$.

$$
+++
$$

4 Eschyle a écrit Les Perses, la plus ancienne des pièces connues du répertoire occidental, dans les suites de la bataille de Salamine en 480 av. J.-C. 5 Anne Ubersfeld, Lire le thêâtre, Paris, Éditions sociales, coll. «Les classiques du peuple. Critique», 1977, p. 176. 6 David Lescot partage cette opinion quand il écrit: «la guerre, davantage qu'une thématique ou que le sujet d'une action, serait la pulsion dramaturgique organisant de l'intérieur les modifications de la forme dramatique canonique». Voir Dramaturgies de la guerre, Paris, Circé, coll. «Penser le thêâtre», 2001, p. 12. 7 Sur cette idée d'obscénité, voir Georges Zaragosa, "La guerre au théâtre, une dramaturgie de l'obscène", L'Information littéraire, vol. LIII, n 3, 2001, p. 3-8. 8 Céline Acker, "La "scène" de la guerre ou la monstration des mécanismes chez Eschyle et Kant», Sens public, n 2, 2005, 19 p., en ligne: http://www.sens-public.org/spip.php?article132 (page consultée le 10 février 2012). 9 Sur cette distinction, voir David Lescot, Dramaturgies de la guerre. 10 Claude Roussin, Marche, Laura Secord!, Montréal, Éditions de l'Aurore, coll. «Entre le parvis et le boxon", 1976, 136 p. 11 Gratien Gélinas, Tit-Coq. Pièce en trois actes, Montréal, Beauchemin, 1950 [créée en 1948], 197 p. ; Marcel Dubé, Un simple soldat. Pièce en cinq actes et quinze tableaux [précédée de] Le temps des lilas. Pièce en trois actes et sept tableaux, Québec, Institut littéraire, 1958 [créée en 1957] p.185-311. 
De ce point de vue, le problème que rencontrent les auteurs dramatiques est double. D'une part, s'agissant de montrer une dramaturgie de l'état de guerre, il leur faut déplacer la tension dramatique du collectif vers l'individuel, vers le personnage qui devra la vivre sur scène, mais, en même temps, résister à une individuation qui aurait pour effet de dissoudre l'effet de guerre en conflit personnel, ce à quoi ne résistait guère la dramaturgie du XIXe siècle ${ }^{12}$. D'autre part, il leur faut inverser cette tension soit en transformant la défaite en victoire - comme l'écrit Jacques Ferron dans Les grands soleils ${ }^{13}$, où il montre comment une défaite peut néanmoins être, dans l'avenir, source du renouvellement de la collectivité —, soit en saisissant les éléments négatifs de la violence exprimée et les séquelles qu'elle laisse dans la vie civile - à l'instar de Wajdi Mouawad, par exemple, dans son traitement des guerres du Moyen-Orient. Les personnages doivent alors entreprendre un voyage, un itinéraire qui leur permet de prendre conscience des incidences que ces guerres ont pu avoir, à leur insu le plus souvent, sur leur identité propre ${ }^{14}$.

Toutefois, Ferron et Mouawad ont en commun de s'intéresser aux guerres civiles et à l'héritage que celles-ci laissent aux générations ultérieures, et l'on peut se demander si le traitement que les auteurs dramatiques accordent aux guerres est déterminé par la nature même de cette guerre. Parle-t-on de la même manière d'une guerre coloniale, d'une guerre civile et d'une guerre mondiale? D'une guerre qui se déroule ici et d'une guerre qui se déroule ailleurs? Chaque guerre a ses enjeux propres, en effet, et chacune reconfigure autrement l'espace de son origine et celui de sa résolution. Je prendrai ici pour exemple la trilogie d'André Ricard qui les aborde toutes: la guerre de Sept Ans, qui s'est soldée par l'abandon de la Nouvelle-France, et dont l'une des batailles déterminantes s'est déroulée à Québec sur les plaines d'Abraham; la guerre civile qu'ont failli devenir les deux rébellions de 1837 et 1838; la Deuxième Guerre mondiale, du débarquement de Normandie aux batailles du Pacifique, à laquelle se sont opposés massivement les Canadiens français, bien que des bataillons de volontaires y aient participé. Écrite et publiée sur vingt-cinq ans, la trilogie, composée de La longue marche dans les Avents ${ }^{15}$ du Tréteau des apatrides ${ }^{16}$ et de Gens sans aveu ${ }^{17}$, porte aussi en elle les marques de l'histoire contemporaine du théâtre

$$
++
$$

12 C'est le cas en particulier de la dramaturgie romantique. Pour le cas québécois, on pourra relire les deux études que j'ai consacrées à Louis Fréchette et à Louis Guyon : «L'art de transformer une défaite en victoire. La représentation des Rébellions dans le thêâtre de Louis Fréchette", Bulletin d’histoire politique, vol. XII, n 1, automne 2003, p. 16-27; "Patriots-on-Broadway : la dramaturgie de Louis Guyon », Études françaises, vol. XXXII, n 3, automne 1996, p. 77-94. 13 Jacques Ferron, «Les grands soleils», Théâtre I, Montréal, l'Hexagone, coll. «Typo», 1990, p. 539-551. 14 Voir le dossier que Jeu. Revue de théâtre consacre à cette question: "Théâtre et guerre », $\mathrm{n}^{\circ}$ 117, 4 trimestre 2005. 15 André Ricard, La longue marche dans les Avents, introduction de Jean Royer, Montréal, Leméac, coll. «Théâtre», 1984, 192 p. Pièce créée au Monument-National le 3 mai 1983 par les élèves finissants de l'École nationale de théâtre, dans une mise en scène de Michelle Rossignol. La pièce est dédiée à Françoise Loranger. Désormais, les références à cet ouvrage seront indiquées par le sigle $L M A$ suivi du folio, et placées entre parenthèses dans le texte. 16 André Ricard, Le tréteau des apatrides ou La veillée en armes. Théâtre. Proposée en lecture publique sur la scène du Théâtre du Nouveau Monde le 3 avril 1995, dans une mise en lecture dirigée par Guy Beausoleil. Coproduction du TNM, du Théâtre d'Aujourd'hui et du CEAD. Désormais, les références à cet ouvrage seront indiquées par le sigle $T A$ suivi du folio, et placées entre parenthèses dans le texte. 17 André Ricard, Gens sans aveu. Théâtre, préface de Guy Beausoleil, Québec, L'instant même, 2008, 209 p. La pièce n'a 
comme celles de la démarche singulière d'un auteur dramatique peu joué et dont l'écriture conquiert progressivement son autonomie en regard de la scène. Ricard nous convie à une relecture de l'histoire du Québec, où chaque guerre reconfigure l'identité nationale.

Aucune de ces guerres n'est représentée directement sur scène. Dans le premier volet, la stratégie de l'auteur est de poser l'action en temps de guerre, mais dans un autre cadre, du côté de ces populations civiles qui, si elles ne se battent pas, n'en vivent pas moins les conséquences du conflit et, parfois malgré elles, doivent prendre position. Dans le deuxième volet, Ricard se pose dans l'instant qui précède l'affrontement, dans un temps immobile, suspendu, comme le temps d'avant l'orage. C'est bien là la structure de l'action dramatique que de montrer la légèreté de l'être qui précède la guerre ou qui en accompagne le début, quand l'issue n'est pas encore décidée. Au contraire, Gens sans aveu, le troisième volet, regarde en arrière. L'action dramatique se situe à l'heure des bilans quand, l'orage apaisé, il reste à prendre la mesure des dégâts et à effectuer le grand nettoyage. L'auteur procède alors à l'envers, en remontant les guerres une à une, comme pour tracer une généalogie qui met en valeur leurs ressemblances, leur identité, plutôt que leurs différences. Cette généalogie est renforcée par une autre généalogie, celle des personnages eux-mêmes, tous descendants les uns des autres et reliés à l'espace originel qu'est le creuset de leur naissance, l'Anse-Jureuse, située au bord du fleuve Saint-Laurent.

\section{LA GUERRE DE SEPT ANS}

La longue marche dans les Avents nous ramène ainsi à la guerre de Sept Ans qui, de 1756 à 1763, oppose les royaumes de France et de Grande-Bretagne sur les théâtres européen et nord-américain. Le titre de la pièce est à prendre dans ses diverses significations. Deux références viennent immédiatement à l'esprit. La «longue marche» rappelle le périple entrepris par l'armée rouge et les communistes, fuyant le régime oppressif et meurtrier du Kouo-min-tang, au début de la Révolution chinoise. Épisode dramatique de l'histoire de cette révolution, à la fois défaite (puisqu'il y a eu fuite) et victoire (puisque s'est constituée là l'unité des forces révolutionnaires), la longue marche est rapidement devenue un symbole de la résistance et un des mythes fondateurs de la société chinoise du xxe siècle. Cette longue marche est "dans les Avents", ce qui n'est pas sans rappeler le temps de l'année qui précède la fête de Noël, temps de l'attente d'une nouvelle naissance qui viendra racheter les péchés du monde. «Avents» est toutefois employé au pluriel et avec la majuscule, ce qui est inhabituel. Il y a peut-être là la mémoire d'un usage plus ancien, contemporain du temps de l'action dramatique ${ }^{18}$, ou encore celui d'un usage régional qui afficherait sa distinction

$$
+++
$$

jamais été jouée à ma connaissance. Désormais, les références à cet ouvrage seront indiquées par le sigle GSA suivi du folio, et placées entre parenthèses dans le texte. 18 L'usage de la majuscule et du pluriel est attesté dès la première édition dans Le dictionnaire de l'Académie française, Paris, Chez la Veuve de Jean-Baptiste Coignard, imprimeur du Roi et de l'Académie française, 1694, p. 624. À l'article «Avent» sont cités les exemples suivants: Les Avents de Noël, C'est aux Avents qu'on a accoustumé de planter. 
d'avec les usages métropolitains, à la manière du vers de Frédéric Mistral, «Lou vènt/Que boufo is Avènt ${ }^{19}$ ". En occitan, en effet, on parle encore des avents au pluriel, comme pour signifier que la naissance est attendue de diverses manières, avec des espérances multiples. Peut-être aussi ce pluriel, associé à la longue marche, désigne-t-il le fait que voici un avent, mais que d'autres, plus tard, adviendront. Quoi qu'il en soit, on notera ici le fait que la guerre, et plus encore la défaite de la France, est présentée comme le point de départ de l'histoire, comme la source d'une vie nouvelle, comme l'espérance d'une naissance, voire d'une renaissance attendue. Il faut en effet disposer de l'ancien monde pour qu'advienne le nouveau.

La pièce se déroule sur deux journées. Au cours de la première, Giraumont et sa femme Marguerite quittent l'Anse-Jureuse pour Québec. Giraumont doit livrer ses peaux et ses récoltes, mais la guerre, ayant fermé l'accès aux territoires de l'Ouest, paralysé les chantiers navals et favorisé le détournement des fonds publics, rend douteux le succès de l'opération. Giraumont constate lui-même qu'« il règne ici une diantre de foire » (LMA, 40). La famille avait pourtant des titres. Des parents sont reçus à la cour par le roi. Un jeune frère, Giraumont de l'Estacade, militaire de carrière, «vient de mériter la Croix de Saint-Louis» (LMA, 27). Une cousine, Geneviève Trémoulé des Barnèches, est illustre: «les paroles qu'elle profère, on se les arrache comme le pain des affamés» (LMA, 27). Giraumont n'en a cure. Seul compte pour lui le commerce: "Ne coule-t-il pas chez vous une seule goutte du sang guerrier de votre famille?» (LMA, 27), demande Marguerite. Les Giraumont vont donc entreprendre le voyage, amenant la bonne, Manette, avec eux puisque la guerre n'a pas que ses mauvais côtés, favorisant les filles à la recherche de maris soldats. On passera par la grève, en raquettes s'il le faut, plutôt qu'en canot à travers les glaces du fleuve. Pas fou, avant d'accepter qu'elle l'accompagne, Giraumont exige de Marguerite qu' elle lui « offre ses faveurs» (LMA, 34). Elle les retient pourtant en garantie: «Menez-moi en vue de la ville: il n'est rien que vous n'obteniez. » $(L M A, 35)$ Le ton est donné. Pièce comique, reposant sur une forte carnavalisation, La longue marche dans les Avents s'inscrit dans le prolongement des pièces précédentes de Ricard où le rire, parfois franc, parfois grinçant, n'en jette pas moins un regard caustique sur le passé politique du Québec ${ }^{20}$.

Le voyage est pourtant difficile, et les Giraumont ne sont qu'à un doigt d'y laisser leur peau (et leurs peaux, bien entendu). Ils sont rescapés par un étrange convoi de dévots, formé du curé Beschefer et de mademoiselle Tranchepain, qui accompagnent vers Québec une jeune Américaine, Elizabeth Osgoode: "Nous l'avons prise aux Trois-Rivières où les Abénaquis qui la retenaient captive ont fini par la rendre [...] car la jeune personne est de conséquence, fille d'un gouverneur de la NouvelleAngleterre.» $(L M A, 63)$ Tous se dirigent vers le relais de Bourg-Joli pour y chercher

$$
+++
$$

19 Frédéric Mistral, Mireille. Poème provençal, édition du centenaire, Paris, Club des libraires de France, 1959, p. 524-525 [texte occitan et texte français en regard]. En français, «is Avènt», qui est un pluriel, est généralement traduit au singulier: «Le vent/Qui souffle à l'Avent». Sur l'usage du nombre en occitan, voir Eduard Koschwitz, Grammaire historique de la langue des Félibres, Genève/Marseille, Slatkine Reprints/Laffitte Reprints, 1973, p. 52. 20 Rappelons ici le titre des précédentes pièces d'André Ricard: La vie exemplaire d'Alcide premier, le pharamineux, et de sa proche descendance, Montréal, Leméac, coll. «Thêâtre », 1973, 174 p. ; La gloire des filles à Magloire, Montréal, Leméac, coll. «Thêâtre», 1975, 156 p. ; Le casino voleur, Montréal, Leméac, coll. «Thêâtre», 1978, 168 p. 
refuge. Giraumont hésite cependant: l'abbé est son ennemi et pourrait demander la saisie de sa récolte, et puis, comme il le rappelle à Marguerite, "c'est la guerre, Madame. Au dernier relais sur la route de Québec, attendez-vous à trouver des soldats » (LMA, 67). Ce soir-là, au relais de Bourg-Joli, Marguerite tient parole et cède joyeusement aux avances de son mari : « Je ne sais, Giraumont, ce qu'il vous est passé par la tête, mais vous avez brûlé de l'encens aux deux autels de l'amour et j'ai comme le sentiment que vous m'avez fait un garçon.» (LMA, 91-92) À la fin de la première journée, la pièce entre donc dans l'avent, cette fois au singulier, car naissance il y aura en effet, tel que le laissait entendre le titre de la pièce.

Reste à disposer du passé, dont il s'agit de faire table rase. Tel sera le propos de la deuxième journée, qui se déroule au couvent de Québec, où les Giraumont retrouvent leur fille Angélique. Autour de ce havre, relativement à l'abri de la société civile, la guerre fait rage. Elle parvient sur scène aux derniers moments, racontée par les religieuses terrorisées, qui entendent le canon et le tocsin alors que les élèves du couvent jouent en représentation «la victoire de Montmorency, où le débarquement fut repoussé avec tant de pertes pour l'envahisseur» (LMA, 160). La population n'a pour résister que les visions de mademoiselle des Barnèches, à qui l'on avait autrefois attribué la victoire de la campagne de fort Outaouagamis et dont on espère qu'elle parviendra encore à « contenir les Anglais et les Bostoniens à nos portes» (LMA, 163). Celle-ci, toutefois, n'en finit plus de prédire le malheur de la Nouvelle-France: «Et toi, peuple à qui rien ne put ployer l'échine, peuple indocile à l'évêque comme à l'intendant, tu connaîtras sur ton arrogance la foulée de l'ennemi.» (LMA, 136) Les faits sont là néanmoins et les nouvelles parviennent une à une aux oreilles des personnages. Des militaires ont été tués, parmi eux, le chevalier Giraumont de l'Estacade, mort au champ d'honneur, et le général de Montclair, enterré dans un trou creusé par un boulet au pied de l'autel. Arrivent encore les blessés, parmi lesquels Picoté Tremblay, qui donne les dernières nouvelles de l'Anse-Jureuse, brûlée pour punir les paysans de leur résistance. Devant un tel désastre, l'abbé Beschefer prend la décision de rentrer en France. Tranchepain elle-même, venue mettre un peu d'ordre dans les finances du couvent, admet qu'il valait mieux ne pas trop espérer en la victoire: «Il fallait les bottes de l'ennemi sur nos places pour que je le comprenne: ce temps est révolu. » (LMA, 188) La foire triomphe de l'ordre.

L'heure des promesses est venue. Manette et Picoté retourneront à l'AnseJureuse : «On partira boire l'eau d'érable à l'Anse-Jureuse. Tout va renaître de sous la cendre.» (LMA, 189) Tranchepain rentrera à Montréal, «la ville que suscita l'inspiration divine et dont le commerce a fait son carrefour » (LMA, 190). Angélique espère que sa mère reçoive son message: «L'enfant dont elle attend la délivrance, mon frère, qu'elle nomme du nom de l'espoir: Jean-Baptiste.» (LMA, 188) La conclusion appartient à Tranchepain, qui annonce: «Femmes! Réjouissons-nous, car nous sommes fécondes. Réjouissons-nous car Noël vient au terme des avents! Réjouissons-nous car nous sommes les trisaïeules de Jean-Baptiste. » (LMA, 191) Se dessine ainsi l'avenir du pays. À la fin de la pièce, quand les canons se sont tus et que la débâcle a emporté le superflu, ne restent en scène que les femmes. Parmi elles, la jeune Elizabeth Osgoode qui refuse de suivre Abercorn, l'émissaire du gouverneur de Boston. La deuxième journée est, somme toute, le récit de leur esseulement, à travers leurs pertes respectives. De cette 
manière, la grossesse de Marguerite apparaît comme une synecdoque, et le fils qu'elle porte, Jean-Baptiste Giraumont, comme l'aïeul d'un peuple nouveau.

\section{L'INSURRECTION DE 1838}

Pourtant, Jean-Baptiste n'est pas le héros du deuxième volet de la trilogie intitulé Le tréteau des apatrides ou La veillée en armes. Privé de ses terres par l'occupant britannique, il s'est, comme son père, réfugié dans les pays d'en haut d'où il exerce son métier d'engagé, un intermédiaire entre les autochtones et les nouveaux marchands britanniques. Nous sommes donc, au départ, dans une pièce qui renvoie aux conséquences de la guerre de Sept Ans, conséquences résumées dans deux mots du titre: «apatrides» et «veillée». Il y a ici l'énoncé d'un vide, d'un manque. Être sans patrie, sans papiers, sans terre, voilà l'héritage, ou plutôt l'absence d'héritage, que JeanBaptiste Giraumont laisse à son descendant, Jean-Eudore Prémont. La pièce s'ouvre sur le retour de Jean-Eudore, lui aussi coureur des bois, commis du nouveau seigneur de l'Anse-Jureuse, McLeod, à qui il vient rendre compte de la liquidation des entreprises du Nord-Ouest. Nous sommes au début du XIXe siècle, et c'est donc à JeanEudore que revient le rôle de traverser les années patriotes, depuis la fondation du premier Canadien, en 1809, jusqu'aux rébellions de 1837 et 1838. L'accompagnent dans cette démarche les deux Bédard, Pierre et Elzéar, le père et le fils, dont les discussions et les affrontements révéleront du même coup les transformations progressives des idées politiques en cours. C'est là le sens du sous-titre, La veillée en armes, qui, comme les avents, désigne un temps d'attente, attente de près de trente ans, qui culminera dans la guerre, guerre civile cette fois.

La structure de la pièce suit la trajectoire de Jean-Eudore au cours de ces années. Préalablement, Ricard nous aura rappelé les circonstances qui ont donné à McLeod la propriété de l'Anse-Jureuse et la manière dont il s'est créé un empire marchand. Marié d'abord à Manette, la bonne des Giraumont, qui n'a jamais été présentée à sa famille écossaise, «rapport qu'[elle] étai[t] pas montrable dans [sa] Gentilly» (TA, 43), il lui donne quatre fils qui, tous, vont mourir à l'une ou l'autre des guerres que conduit l'Empire britannique. C'est donc à ses filles que Manette espère rendre l'Anse-Jureuse. La deuxième femme de McLeod est autochtone et elle accouche de trois fils qui gèrent les affaires de l'Ouest, mais que McLeod préfère ne pas voir trop souvent en ville: «Race forte que celle des métis. Mais méprisable comme tous les sang-mêlé. Qui aurait obéi à des bâtards ? À des Bois-brûlés ? » (TA, 57) Aussi est-ce le petit-fils de Manette, William McLaughlin, qui héritera de la seigneurie. Une troisième épouse, Edwina, est anglophone et protestante. À McLeod elle donne deux filles, dont Anne, qui s'occupe de son père devenu vieux et qui gère le domaine pour son neveu William, toujours absent: «Les fermes, puisque leurs fils sont militaires, ils [les propriétaires] les confient à la branche mineure de la famille.» (TA, 186) Contrairement à ses demi-frères métis qui protestent et s'insurgent, Anne réagit en «bon[ne] domestique [attachée] aux intérêts du maître» (TA, 187). Ainsi, les filles de Manette ont épousé des Anglais, selon le désir de leur père, alors que les filles d'Edwina ont épousé des Canadiens. Et l'on comprend pourquoi le fils d'Anne, Duncan Simard, a rejoint le parti patriote: «La 
branche pauvre de la famille est destinée à servir et à parler français comme l'autre à accumuler et à reconnaître une appartenance anglaise.» (TA, 187) On le voit, le torchon brûle déjà chez les McLeod où trois familles, trois peuples, se disputent le territoire et l'héritage, séparés et opposés les uns aux autres par la volonté certains diraient l'incurie du patriarche. À partir de ce conflit familial, Ricard fait l'exposé des conditions socioéconomiques de la Province of Quebec, sous la domination britannique. Mais l'on sent bien que le monde change à mesure que la tension monte entre McLeod, ses fils et ses petits-fils.

Remercié de ses services et mis au chômage à son retour de l'Ouest, JeanEudore reste indifférent aux causes et aux enjeux qui ont mené à son congédiement. Réfugié au bordel, il se trouve malgré lui engagé dans une rixe qui se termine par la mort d'une des filles, Palmyre, accidentellement tuée. Il est alors condamné à mort et enfermé à la prison de Québec dans une cellule où se trouve déjà Pierre Bédard. Il lui reste cinq heures à vivre. Il en profitera pour faire l'apprentissage de la politique, prendre connaissance de la nouvelle configuration de la sphère publique, débattre de la légitimité de l'Assemblée législative et de la représentation électorale. Le contraste entre la vie des McLeod et celle de Pierre Bédard est saisissant. Ceux-là vivent toujours à l'heure de la Province of Quebec, dans un régime seigneurial fondé sur l'exploitation de la terre et des autres ressources naturelles (notamment les fourrures). Bédard, quant à lui, est déjà dans le Bas-Canada, un monde bourgeois fondé sur la compétence, le mérite, le travail, l'industrie et la politique. Jean-Eudore n'est pas convaincu.

L'entrée en scène de Peggy Hammerslough constitue une heureuse diversion. Celle-ci collectionne les portraits de condamnés à mort saisis trois jours avant leur exécution : «une fois qu'il aura chanté son propre Requiem, vous verrez [le] condamné fléchir le torse vers le foyer de l'angoisse [... .]. Les yeux, la bouche s'aigrir d'une mortelle désespérance» $(T A, 75)$. Épouse de William McLaughlin, elle appartient à un monde dégagé de toute contingence matérielle, voué à la culture des arts et des sciences comme autant d'artefacts d'un monde révolu, comme le sera, dans la deuxième partie de la pièce, ce lord Lambeth, clone du Durham de l'histoire. Délicieusement décadents, ces Britanniques d'Ancien Régime continuent de séduire Jean-Eudore qui reste indifférent aux enjeux politiques qui l'entourent. Toutefois, le choléra sévit et les prisonniers qui acceptent d'enterrer les morts bénéficient d'un sursis. Jean-Eudore va donc enterrer les corps.

Entre la première et la deuxième partie de la pièce, des années se sont écoulées. Jean-Eudore a été fossoyeur puis baleinier, gabier, secrétaire de lord Lambeth, et sa sentence a été reportée. Il est revenu chez la mère Maquerelle et reçoit la visite du député Bédard de la Chambre d'Assemblée du Bas-Canada. C'est cette fois Elzéar, fils de Pierre, qui lui remet son passeport en lui rappelant que sa sentence demeure "exécutoire selon le bon plaisir de Sa Majesté» $(T A, 155)$ et qu'il est en sursis. Il l'invite alors à prendre parti pour les Patriotes. Jean-Eudore reprend plutôt son poste auprès de lord Lambeth, qui s'apprête à rédiger son rapport... à l'avance. Il y a là une suave prolepse qui permet à Jean-Eudore d'imaginer l'avenir du pays et d'en informer Elzéar Bédard: «Les [Patriotes] plus résolus de l'état-major chercheront refuge dans les villages où la fièvre nationaliste a atteint son comble. Ces quelques poches de résistance 
armée serviront de prétexte à instaurer le régime militaire ; et surtout, à faire de terribles exemples.» (TA, 211)

Il n'y a pas d'action dramatique dans Le tréteau des apatrides, qui relève plutôt du registre du théâtre épique, mettant en scène un personnage qui n'assume jamais la position de sujet de sa propre destinée et qui apparaît comme l'acteur interprétant le rôle que lui ont réservé les occupants britanniques : engagé, condamné, secrétaire. De son point de vue, la « veillée en armes » n'existe pas. Il lui faudra d'abord prendre conscience de l'état des faits, ce qui se produit progressivement au contact de ceux qui ont déjà entrepris la guerre : les deux Bédard, père et fils - bien que le père se soit finalement rallié à la cause britannique - , mais aussi Duncan Simard, fils d'Anne McLeod. Il lui faudra surtout transformer son état d'apatride en malaise. Le souvenir de Palmyre, qui surgit à la fin de chacune des deux parties de la pièce, permet de mesurer cette lente prise de conscience. La première fois, elle demande: "Pourquoi es-tu devenu un nomade, Jean-Eudore, et pourquoi étais-je obligée de gagner ma pitance comme tu as vu? » $(T A, 127)$ La seconde fois, elle "dépose un capot d'habitant devant Elzéar» $(T A, 212)$ et remet à Jean-Eudore une pochette de "poussière du village d'où [elle] vien[t]». Puis elle prédit: "Ils seront quelques-uns vêtus comme tu l'es qu'on débusquera à la baïonnette dans l'église en flammes ou dans le cimetière. Et on en accrochera bien une douzaine au bout d'une corde.» (TA, 212)

\section{LE DÉBARQUEMENT DE NORMANDIE}

Plus d'un siècle et demi sépare le temps dramatique du Tréteau des apatrides de celui de Gens sans aveu, dernier volet de la trilogie. Nous sommes en 1996. Dans une maison de retraite réservée aux vétérans, de jeunes gens offrent une représentation dramatique à l'occasion des festivités de la Saint-Jean-Baptiste. Cette représentation emprunte à la vie du brigadier Lanteigne, qui a publié un ouvrage intitulé, précisément, Gens sans aveu. Dans ce livre, Lanteigne ne s'est pas contenté de raconter la guerre - ici la Seconde Guerre mondiale, depuis le débarquement de Normandie. Il a une opinion et ne la cache pas. Sa thèse générale est, comme la résume efficacement le préfacier Guy Beausoleil, que «les peuples assujettis, subalternes - les Canadiens français, entre autres - sont destinés à l'opprobre et à la proscription après avoir servi en première ligne au cours des conflits militaires opposant les nations dominantes » $(G S A, 25)$. Il s'agit donc d'un « livre accusateur» (GSA, 39), d'un témoignage que les personnages eux-mêmes rapprochent de Nègres blancs d'Amérique de Pierre Vallières $(G S A$, 57). La représentation dramatique qui se prépare est un hommage à un pensionnaire de l'hôpital des vétérans en même temps qu'un réquisitoire pour que ceux-ci obtiennent de meilleures conditions de vie : "Ça prenait la bisbille au sujet du livre de Lanteigne pour qu'on nous sorte des boules à mites. » (GSA, 72)

Car «bisbille» il y a, et la référence à Vallières n'est pas fortuite. Récit de guerre, le livre de Lanteigne l'est, oui, mais c'est d'abord un récit de vie écrit dans de troubles circonstances. Quelque temps auparavant, un documentaire télévisé sur la vie de

Lanteigne a été retiré des ondes à la suite, paraît-il, de plaintes reçues, et ce retrait a lui-même créé un certain remous. Des étudiants sont alors venus pour enregistrer 
l'histoire de sa vie et, en relisant le tout, Lanteigne s'est intéressé à l'Anse-Jureuse, son lieu d'origine, puis il a écrit son livre. Ce sont les réservistes du régiment PapineauNelson, dont le brigadier Lanteigne est le commandant honoraire, qui vont jouer la pièce devant un parterre composé de représentants du gouvernement et du clergé. Prégent, responsable du protocole, s'inquiète de cette représentation. Après tout, les célébrations de la Saint-Jean-Baptiste rappellent toujours un peu les émeutes de 1968, et on commémore à peu près en même temps le 160 anniversaire des rébellions de 1837 et 1838 .

La pièce est ainsi construite sur plusieurs plans. À l'avant-scène se déploient les conditions de la représentation. Nous sommes à l'hôpital des vétérans, qui s'apprête à recevoir des invités de marque. Priment le protocole et les inquiétudes de Prégent. À l'arrière-plan, la représentation elle-même, tirée du livre de Lanteigne, remonte le fil de sa vie pour montrer comment il est devenu l'homme qu'il est. Les moments clés de cette histoire nous ramènent aux deux volets précédents de la trilogie, puisque Lanteigne est lui aussi le descendant des Giraumont de l'Anse-Jureuse. Un troisième plan présente une image relativement fixe, une pietà, figure forte qui traverse le temps et l'espace. Toutefois, cette pietà toujours en scène est reconfigurée par chaque guerre, les grandes comme les petites: une femme tient un jeune soldat ensanglanté sur ses genoux recouverts de l'unifolié $(G S A, 35)$; une femme indienne tient un Inuit sur ses genoux (GSA, 134); une femme tient Lanteigne, recouvert du fleurdelisé, sur ses genoux (GSA, 192).

Il y a donc deux Lanteigne: celui qui, depuis l'âge de seize ans, s'est battu à chaque guerre au service de la puissance britannique, apatride comme ses aïeux dépossédés de leurs terres, toute une filiation formée de mercenaires à la solde des marchands puis des soldats, gens sans aveu, c'est-à-dire « [g]ens sans profession, oui, jusqu'à ce qu'on leur mette un fusil dans les mains» (GSA, 60). Celui-là était bigame, ayant d'abord épousé Paulette en Angleterre, à la veille de son emprisonnement au Stalag, puis Jacinthe à l'Anse-Jureuse, à son retour des guerres du Pacifique, et il souffre visiblement d'un syndrome de stress post-traumatique que révèlent ses accès de colère. Puis il y a l'autre Lanteigne, celui qui remonte le fil de l'histoire pour mieux comprendre la généalogie, la sienne d'abord, depuis les Giraumont, puis une autre, «celle de la guerre, où il décline aussi ses antécédents ( $G S A, 141)$. Le premier est le petit-fils de Xavier Lanteigne, qui avait servi contre Louis Riel dans la répression des rébellions de l'Ouest; le second se réclame désormais d'un autre aïeul, Jean-Eudore Prémont, mort avec Jean-Olivier Chénier en proclamant la république.

\section{RELIRE JACQUES FERRON}

Entre les avents, qui annoncent une naissance, et le temps des vétérans, qui se clôt sur le suicide du vieux colonel Bradshaw, près de deux siècles et demi se sont écoulés d'une fresque historique qui forme comme "une légende des siècles ${ }^{21}$ ", selon le mot de Guy

$$
+++
$$

21 «Arrimant son récit-fleuve à quelques dates nodales de l'histoire du Québec, le dramaturge édifie une légende des siècles toute personnelle», écrit Guy Beausoleil dans la préface de Gens sans aveu, p. 11-12. L'auteur souligne. 
Beausoleil, où chaque chant évoque une des trois grandes époques de l'histoire du Québec: la Nouvelle-France, le Bas-Canada et la province de Québec. Il s'agit de saisir chacune de ces époques au moment de son dépérissement, quand l'équilibre des forces se rompt, entraînant la radicalisation du conflit et, le cas échéant, la lutte armée. Aussi les forces dominantes apparaissent-elles toujours délicieusement décadentes, depuis les Giraumont eux-mêmes, parents de la visionnaire Geneviève Trémoulé des Barnèches aux prises avec ses démons, jusqu'à l'abbé Beschefer, méprisant comme aucun autre envers les habitants du pays; depuis le vieux McLeod, qui n'en finit plus de pleurer sur ses épouses décédées, la première surtout, et l'étrange couple formé par Liébert, l'artiste français spécialisé dans les portraits de condamnés à mort, et Peggy Hammerslough qui les commandite, jusqu'au lord Lambeth, profitant de son serviteur acheté en Inde, et à son épouse, l'éthéromane lady Henricke; depuis Giroux, l'attaché politique du ministre de la Guerre, venu rendre publique l'annonce du déménagement de l'hôpital des vétérans, qu'il veut voir toujours plus loin de la civilisation, jusqu'à Monseigneur Rancourt, avocat principal dans la cause de béatification de la vénérable Geneviève Trémoulé des Barnèches, en passant par les vétérans eux-mêmes, âgés et mal en point.

Cette décadence un peu morbide, qui caractérise la plupart des personnages anglais, ecclésiastiques ou militaires, n'est pas sans rappeler la manière de Jacques Ferron, et on pourrait ainsi lire la trilogie de Ricard comme une réécriture des Grands soleils, dont la mémoire resurgit à chaque scène. Cette mémoire se trouve d'abord dans l'économie générale du propos, qui construit une société québécoise pluraliste, composée d'hommes et de femmes, de francophones et d'anglophones, de blancs et d'autochtones, réunis par leur condition, qui promeut une forme de solidarité entre les apatrides et les gens sans aveu de tous les pays et de toutes les époques, comme autant de victimes civiles des guerres, et qui affirme la victoire des fils contre leurs pères comme une nécessaire projection dans l'avenir. Cette mémoire se lit également dans l'écriture, où se retrouvent divers procédés de carnavalisation, en particulier dans la construction des noms propres (Trémoulé des Barnèches, Tranchepain, Beschefer) et des situations (depuis la joyeuse séance de copulation de La longue marche dans les Avents jusqu'à la gigue de Monseigneur Rancourt qui clôt Gens sans aveu). Nulle part elle n'est aussi apparente que dans le personnage d'Elizabeth, fille du gouverneur de Boston, enlevée et élevée par les Abénaquis, réfugiée chez les ursulines, qui semble elle-même une sorte d'ancêtre du personnage des Grands soleils de Ferron, cette autre Elizabeth tout aussi anglaise mais venue on ne sait d'où, élevée par les ursulines et solidaire des Patriotes.

Toutefois, si la référence à Ferron s'impose ici, c'est surtout que, parce qu'il s'agit de thêâtre historique, d'un théâtre qui interroge l'histoire du Québec, l'œuvre de Ricard se trouve à s'insérer dans une série de pièces qui se répondent l'une à l'autre autour de quelques grandes problématiques récurrentes. Ainsi, dans Les grands soleils, Ferron découd la vision des rébellions de 1837 et 1838 proposée par Louis Fréchette dans Félix Poutré, remettant en question le personnage autant que l'orientation conservatrice de la conclusion qui soumet le fils à la volonté du père. Ce faisant, Ferron projette le personnage de François Poutré, fils de Félix, dans un avenir utopique qui, à terme, transforme la défaite des rebelles en victoire de la nation québécoise désormais constituée. Pour y parvenir, il aura néanmoins fallu que le personnage entreprenne un voyage 
initiatique autour du monde, au cours duquel il tisse des solidarités multiples avec les autres victimes civiles de la guerre: «il était à Saint-Eustache, il était dans tous les fours crématoires, à Hiroshima, à Dresde, à Hanoi ${ }^{22}$ ». Ainsi la pièce de Ferron débordet-elle du temps des seules Rébellions, vécues au début du XIX siècle, pour déployer une réflexion sur l'identité collective en tant qu'elle se construit dans un rapport à l'Autre, rapport conflictuel qui s'exprime dans la lutte armée, mais aussi rapport de solidarité qui se découvre dans les pérégrinations du personnage de François Poutré.

«Tout peuple apatride sur sa propre terre/est un peuple de gens sans aveu/ exclu de la mise en valeur/des conflits même où l'histoire/le tiendra parqué/jusqu'à la reconquête de ses droits.» (GSA, 194) Telle est la conclusion du livre de Lanteigne, et telle est aussi la conclusion de la trilogie d'André Ricard, sur cette thèse qui se présente sous la forme d'un aphorisme. Ce n'est donc pas le conflit des frontières qui est l'enjeu de la trilogie d'André Ricard, mais, comme chez Ferron, l'identité nomade d'un peuple sans pays, voué à la dispersion géographique. S'il s'y trouve néanmoins toujours un conflit d'espace, celui-ci est dans l'opposition entre les lieux clos (le couvent, le bordel, la prison, la maison de repos) et les déplacements (la longue marche des Giraumont, les pérégrinations de Jean-Eudore, les guerres de Lanteigne) qui exposent les personnages au risque d'une altération de soi. Chaque fois, au bout du voyage, le personnage est confronté à la guerre. Le retour à l'Anse-Jureuse, fût-il symbolique, crée alors un point de fuite qui permet au personnage de se reconstituer en sujet de son histoire. Ainsi, les guerres ne dépossèdent pas les personnages du territoire, de ce territoire qui n'a jamais été le leur, mais elles transforment leur identité. En effet, « [l]'une après l'autre, les guerres donnent naissance à une société nouvelle» (GSA, 141), explique Brunelle, faisant écho à Peggy Hammerslough qui affirmait que la guerre de Sept Ans avait été «comme une deuxième naissance pour la colonie» (TA, 77). La trilogie offre ainsi une vision dialectique de l'histoire qui pose le conflit comme constituant nécessaire ou en tout cas inévitable de toute société; la résolution du conflit n'engendre pas la paix, mais une nouvelle contradiction, un nouveau conflit, une nouvelle guerre. Ainsi, à l'histoire close et utopique de Ferron, Ricard oppose la dialectique historique. S'il reste une part d'utopie dans l'écriture d'André Ricard, elle se profile dans le découpage d'un village à défendre, d'une terre à occuper, d'une patrie à définir qui, bien que jamais atteint, n'en représente pas moins un rêve, un horizon à construire. De ce point de vue, le dernier volet de la trilogie, Gens sans aveu, parait porter une visée testamentaire, celle de Lanteigne au soir de sa vie bien sûr, mais sans doute aussi celle de Ricard, qui engage une lecture généalogique de l'histoire, comme en un témoignage destiné désormais à ceux qui viendront. Hors de cette généalogie, la trilogie ne peut avoir de veritable clôture, puisque la patrie reste encore à construire, ad infinitum.

Le thêâtre a longtemps conçu l'action dramatique comme un conflit entre deux forces ou personnages. Sur scène, et quelles qu'en soient les causes immédiates, ce conflit vise l'occupation de l'espace scénique. Du coup, dans cette conception aristotélicienne du thêâtre, la logique individuelle du conflit devient indépassable. Contre

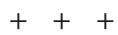


cette individuation s'impose alors, pour parler de la guerre, le choix du registre épique, qui décompose l'action dramatique en séquences plus proches de la logique narrative, qui dès lors devient une structure argumentative. Par là d'abord, la dramaturgie de la guerre devient une dramaturgie de l'état de guerre. Écrite et publiée sur vingt-cinq ans, la trilogie reflète aussi une démarche d'écriture, encore dramatique dans La longue marche dans les Avents, dont le comique rappelle certaines scènes de La vie exemplaire d'Alcide Premier le pharamineux ou du Casino voleur, résolument épique dans Le tréteau des apatrides, où le personnage principal doit apprendre à devenir le sujet de sa destinée, plus fortement épicisée ${ }^{23}$ dans Gens sans aveu, où la représentation dramatique mise en abyme, relevant elle-même du registre épique, est médiatisée par le livre de Lanteigne, que résume fréquemment Brunelle au bénéfice de Prégent... et du lecteur/spectateur. Toutefois, dans La longue marche dans les Avents, le caractère strictement dramatique de l'action était déjà sapé par l'indifférence des personnages à la guerre se déroulant autour d'eux (et hors scène); cette guerre-là, découvrent-ils eux-mêmes, n'est pas la leur bien qu'elle révèle les conditions de leur existence comme peuple. Dans Le tréteau des apatrides, la prise de conscience du personnage principal survient à la toute fin de la pièce comme l'issue d'un long cheminement. Dans Gens sans aveu, la révolte de Lanteigne a déjà eu lieu. Aussi la représentation dramatique est-elle mise en abyme et médiatisée par le livre éponyme. La figure du livre, inscrite au terme de cette dramaturgie de l'état de guerre, accentue la mise à distance de l'événement historique, et montre l'aboutissement de la réflexion et de la révolte. En même temps, ce livre-là devient la source d'un autre geste, celui accompli par les plus jeunes qui, à travers le théâtre, réincarnent, réitèrent la révolte. La prise de conscience est donc à degrés multiples: elle est celle du vieux Lanteigne d'abord, celle des jeunes acteurs ensuite et elle se prolonge encore chez le spectateur/lecteur. Il n'y a pas de suspense dans la trilogie, rien qui soit vraiment inattendu. La guerre a été vécue dans l'histoire; l'issue en est trop connue pour qu'il vaille la peine d'y revenir. Reste à voir comment sont arrivés là ceux et celles qui l'ont faite, quels bilans ils peuvent en tirer et quelle sera la mémoire de ces événements. Chez Ferron, la victoire était celle du pays tout entier ( «Le pays est à nous ${ }^{24}$ »), dans la mémoire qui constitue la nation; chez Ricard, plus modestement peut-être, elle est dans cette conviction que la force, qui trace les frontières, ne dispose pas des peuples.

23 À la différence de l'épique brechtien, où la structure en tableaux découpe un temps proche de celui du récit, mais maintient néanmoins l'évênement historique sur scène, le thêâtre "épicisé» renvoie l'action dans un temps coupé du présent dramatique et les personnages racontent alors une histoire survenue avant le début du drame. Sur cette notion, voir Laurence Barbolosi et Muriel Plana, «Épique/épicisation », Jean-Pierre Sarrazac (dir.), Lexique du drame moderne et contemporain, Belval, Circé, coll. "Circé poche», 2005, p. 74. 24 Jacques Ferron, Les grands soleils, p. 544. La phrase est reprise telle quelle dans Le tréteau des apatrides, mais elle est énoncée par McLeod (TA, 39). 